\title{
J-RINGS OF CHARACTERISTIC TWO THAT ARE BOOLEAN
}

\author{
D. J. HANSEN and JIANG LUH \\ Department of Mathematics \\ North Carolina State University \\ Raleigh, North Carolina 27695-8205, U.S.A.
}

YOUPEI YE

East China Institute of Technology

Nanjing, China

(Received Ju1y 27, 1993)

\begin{abstract}
This paper is concerned with determining all integers $n$, with $n \geq 2$, such that if $R$ is a ring having the property that $x^{n}=x$ and $2 x=0$ for each $x \in R$, then $R$ is boolean. The solution to the above problem extends previous results obtained by Shiue and Chao in [5] and that of MacHale in [4].
\end{abstract}

KEY WORDS AND PHRASES. J-ring, boolean ring. 1992 AMS SUBJECT CLASSIFICATION CODES. 16A38.

1. INTRODUCTION.

A ring $R$ is called a J-ring if there exists an integer $n \geq 2$ such that $x^{n}=x$ for each $x \in R$. It is well known that a J-ring is commutative, see [3].

Shiue and Chao showed in [5] that if $R$ is a J-ring, where $n=2^{q(m+1)}+2^{m}$, with $1 \leq q$ and $1 \leq m$, then it is the case that $R$ is of characteristic two and, in addition, $x^{2}=x$ for each $x \in R$, that is, $R$ is boolean. Recently, MacHale proved that if $R$ is a ring of characteristic two and $n$ is a nonnegative integer such that $x^{2^{n}+1}=x$, for each $x \in R$, then $R$ is also boolean. In this paper, we will extend both of the above results by determining all integers $n$, with $n \geq 2$, such that if $R$ is a ring having the property that $x^{n}=x$ and $2 x=0$, for each $x \in R$, then $x^{2}=x$ for each $x \in R$. It should be noted that, in a related paper, Batbedat [2] used sheaf theory to obtain some structure theorems for a ring $R$ satisfying $a^{n+1}=a$ for each $a \in R$. His results were used to determine all values of $n \leq 50$ for which $R$ is boolean.

2. A PRELIMINARY RESULT.

THEOREM 1. Let $n$ denote an integer $\geq 2$. Then $2^{t}-1 \nmid n-1$ for each integer $t \geq 2$ if and only if for each ring $R$ such that $x^{n}=x$ and $2 x=0$ for each $x \in R$ implies that $R$ is boolean.

PROOF. Suppose $2^{t}-1 \mid n-1$ for some integer $t \geq 2$. Let $R$ denote the Galois field GF $\left(2^{t}\right)$. If $x \in R$, with $x \neq 0$, then $x^{2^{t}-1}=1$ and thus $x^{n-1}=1$ since $2^{t}-1 \mid n-1$. Hence $x^{n}=x$. Since $0^{n}=0$, we thus have that $x^{n}=x$ for each $x \in R$. It is well known that $\operatorname{GF}\left(2^{t}\right)$ is of characteristic 2. Consequently, $R=G F\left(2^{t}\right)$ is a ring such that $x^{n}=x$ and $2 x=0$ for each $x \in R$, however $R$ is not boolean. 
Next assume that $2^{t}-1 \nmid n-1$ for each $t \geq 2$. Suppose to the contrary that there exists a nonboolean ring $R$ such that $x^{n}=x$ and $2 x=0$ for each $x \in R$. Then there exists an $a \in R$ such that $a^{2} \neq a$. Consider $\langle a\rangle$, the subring of $R$ generated by $a$. First, note that $\langle a\rangle$ is finite and commutative. Also, $\langle a\rangle$ is semi-simple since, for each $x \in\langle a\rangle, x^{n-1}$ is idempotent and the Jacobson radical of $\langle a\rangle$ does not contain non-zero idempotent elements. Hence, by the Wedderburn-Artin theorem, $\langle a\rangle$ is a direct sum of finitely many Galois fields of characteristic 2 , say, $\langle a\rangle=\sum_{i=1}^{m} G F\left(2^{t_{\cdot}}\right)$. Clearly, $2^{t_{1}}-1 \mid n-1$ for $i=1,2, \cdots, m$ and thus $t_{t}=1$ for $i=1,2, \cdots, m$ since $2^{t}-1 \nmid n-1$ for each $t \geq 2$. Hence $a^{2}=a$ and this is a contradiction. Therefore if $R$ is a ring of characteristic two such that $x^{n}=x$ for each $x \in R$, then $R$ is boolean.

\section{A SPECIAL CLASS OF MATRICES.}

Let $k$ denote an integer $\geq 2$. For each such $k$, define the matrix $M_{k}$ to be the matrix with $k$ columns whose rows are of the form $\left[k-t_{1}, k-t_{2}, \cdots, k-t_{k}\right]$, where each $t_{1}, t_{2}, \cdots, t_{k}$ is a non-negative integer such that $t_{1} \geq t_{2} \geq \cdots \geq t_{k}$ and $2^{t_{1}}+2^{t_{2}}+\cdots+2^{t_{k}}=2^{k}$. Furthermore, if $r=\left[k-t_{1}, k-t_{2}, \cdots, k-t_{k}\right]$ and $r^{\prime}=\left[k-t_{1}^{\prime}, k-t_{2}^{\prime}, \cdots, k-t_{k}^{\prime}\right]$ are two rows in $M_{k}$, then $r$ is a above $r^{\prime}$ if and only if either $k-t_{1}<k-t_{1}^{\prime}$ or $k-t_{i}=k-t_{i}^{\prime}$ for $i=1,2, \cdots, m$ and $k-t_{m+1}<k-t_{m+1}^{\prime}$.

The following lemma and theorem will give an inductive method for constructing the above type of matrices.

LEMMA 2. Let $k$ denote an integer $\geq 2$. Let each of $t$ and $t_{1}, t_{2}, \cdots, t_{k}$ denote a nonnegative integer such that $2^{t_{1}}+2^{t_{2}}+\cdots+2^{t_{k}}=2^{t}$, where $t_{1} \geq t_{2} \geq \cdots \geq t_{k}$. Then $t_{k-1}=t_{k}$.

PROOF. Clearly $t>t_{i}$ for $i=1,2, \cdots, k$. From $2^{t_{1}}+2^{t_{2}}+\cdots+2^{t_{k}}=2^{t}$, we obtain $2^{t_{1}-t_{k}}+2^{t_{2}-t_{k}}+\cdots+2^{t_{k-1}-t_{k}}+1=2^{t-t_{k}}$. Since $2^{t-t_{k}}$ is even, then at least one of $t_{i}-t_{k}, 1 \leq$ $i \leq k-1$, is zero, say, $t_{j}-t_{k}$. Then $t_{j}=t_{k}$ which implies that $t_{k-1}=t_{k}$ since $t_{j} \geq t_{k-1} \geq t_{k}$.

LEMMA 3. Let $k$ denote an integer $\geq 2$. If $l$ is an integer, with $2 \leq l \leq k$, and if each of $t_{1}, t_{2}, \cdots, t_{l}$ is a nonnegative integer such that $2^{t_{1}}+2^{t_{2}}+\cdots+2^{t_{l}}=2^{k}$, then $t_{i}>0$ for all $i, 1 \leq i \leq l$.

PROOF. The proof is by induction on $k$. Let $S$ denote the set such that $k \in S$ if and only if $k \geq 2$ and $2^{t_{1}}+2^{t_{2}}+\cdots+2^{t_{1}}=2^{k}$ implies that $t_{i}>0$ for $1 \leq i \leq l$, where $2 \leq l \leq k$. For $k=2$, the only equation, since $l=2$, is $2^{t_{1}}+2^{t_{2}}=2^{2}$ and this implies that $t_{1}=t_{2}=1$. Thus $2 \in S$. Let $k \in S$. Next, let $2 \leq l^{\prime} \leq k+1$ and suppose $t_{1}^{\prime} \geq t_{2}^{\prime} \geq \cdots \geq t_{l^{\prime}}^{\prime} \geq 0$ such that $2^{t_{1}^{\prime}}+2^{t_{2}^{\prime}}+\cdots+2^{t_{{ }^{\prime}}^{\prime}}=2^{k+1}$. Now, suppose there exits a $t_{i}^{\prime}$ which is zero. Then either all of the $t_{1}^{\prime}$ 's are zero, and thus $l^{\prime}$ would be even in that case or there exists a $j$ such that $t_{j}^{\prime}>0$ with $t_{j+1}^{\prime}=0$ and this would imply that there exists again an even number of $t_{i}^{\prime}$ 's equal to zero. Hence, in either ease, we can group the $2^{o}$ 's in pairs and obtain either $\left(2^{o}+2^{o}\right)+\left(2^{o}+2^{o}\right)+\cdots+\left(2^{o}+2^{o}\right)=2^{k+1}$ or $2^{t_{1}^{\prime}}+2^{t_{2}^{\prime}}+\cdots+2^{t_{j}^{\prime}}+\left(2^{o}+2^{o}\right)+\cdots+\left(2^{o}+2^{o}\right)=2^{k+1}$. Since $2^{o}+2^{o}=2$, we have either $2^{1}+2^{1}+\cdots+2^{1}=2^{k+1}$ or $2^{t_{1}^{\prime}}+2^{t_{2}^{\prime}}+\cdots+2^{t^{\prime}}+2^{1}+\cdots+2^{1}=2^{k+1}$ and this gives, on dividing both sides by 2 , either $2^{o}+2^{o}+\cdots+2^{o}=2^{k}$ or $2^{t_{1}^{\prime}-1}+2^{t_{2}^{\prime}-1}+\cdots+2^{t_{j}^{\prime}-1}+2^{o}+\cdots+2^{o}=2^{k}$. Note that the number of terms on the left in either equation is now $\leq k$. Hence we have arrived at a contradiction since $k \in S$ implies that all of the exponents in either equation must be positive. Therefore $k+1 \in S$ and this completes the induction argument.

COROLLARY 4. $M_{2}=[1,1]$.

PROOF. For $k=2$, we consider $2^{t_{1}}+2^{t_{2}}=2^{2}$. From Lemma 2, we have that $t_{1}=t_{2}$. Thus $2^{t_{1}+1}=2^{2}$ which implies that $t_{1}=1$. Since $t_{2}=t_{1}$, we have that $t_{2}=1$. Hence $M_{2}=$ $\left[k-t_{1}, k-t_{2}\right]=[2-1,2-1]=[1,1]$. 
THEOREM 5. Suppose $M_{k}=\left[s_{i j}^{(k)}\right]$. Then the rows of $M_{k+1}$ are precisely the rows obtained from the rows of $M_{k}$ by replacing one entry $s_{i j}^{(k)}$ by the $1 \times 2$ matrix $\left[s_{i j}^{(k)}+1, s_{i j}^{(k)}+1\right]$, and following this by a suitable rearrangement of the entries.

PROOF. First, we will show that a row obtained from the $i^{\text {th }}$ row of $M_{k}$ by replacing the entry $s_{i j}^{(k)}$ by $\left[s_{i j}^{(k)}+1, s_{i j}^{(k)}+1\right]$ is, follwed by a suitable rearrangement of the entries, a row in the matrix $M_{k+1}$, that is, $\left[s_{i 1}^{(k)}, \cdots, s_{i, j-1}^{(k)}, s_{i j}^{(k)}+1, s_{i j}^{(k)}+1, s_{i, j+1}^{(k)}, \cdots, s_{i k}^{(k)}\right]$ follwed by rearranging the numbers in ascending order will be a row in $M_{k+1}$. To see that this is the case, consider, from the definition of $M_{k+1}$, the $\operatorname{sum} \sum_{q=1}^{j-1} 2^{k+1-s_{i q}^{(k)}}+2^{k+1-\left(s_{i}^{(k)}+1\right)}+2^{k+1-\left(s_{i j}^{(k)}+1\right)}+\sum_{q=j+1}^{k} 2^{k+1-s_{i q}^{(k)}}=\sum_{q=1}^{j-1} 2^{k+1-s_{i q}^{(k)}}$ $+2^{k+1-s_{i j}^{(k)}}+\sum_{q=j+1}^{k} 2^{k+1-s_{i q}^{(k)}}=\sum_{q=1}^{k} 2^{k+1-s_{i q}^{(k)}}=2 \sum_{q=1}^{k} 2^{k-s_{i q}^{(k)}}=2 \cdot 2^{k}=2^{k+1}$. Hence, from the definition of $M_{k+1}$, we have confirmed what we stated above.

Next, we need to show that each row of $M_{k+1}$ is obtained from a certain row of $M_{k}$ by the above described replacement. Let $r_{i}=\left[s_{i 1}^{(k+1)}, s_{i 2}^{(k+1)}, \cdots, s_{i, k+1}^{(k+1)}\right]$ be the $i^{\text {th }}$ row of $M_{k+1}$. Then, from the definition of $M_{k+1}$ and Lemma $3, \sum_{q=1}^{k+1} 2^{k+1-s_{i q}^{(k+1)}}=2^{k+1}$ and $1 \leq s_{i 1}^{(k+1)} \leq s_{i 2}^{(k+1)} \leq$ $\cdots \leq s_{i k}^{(k+1)} \leq s_{i, k+1}^{(k+1)} \leq k$. By Lemma $2, k+1-s_{i k}^{(k+1)}=k+1-s_{i, k+1}^{(k+1)}$ or $s_{i k}^{(k+1)}=s_{i, k+1}^{(k+1)}$. Thus $2^{k+1}=\sum_{q=1}^{k+1} 2^{k+1-s_{i q}^{(k+1)}}=\sum_{q=1}^{k-1} 2^{k+1-s_{i q}^{(k+1)}}+2^{k+2-s_{i k}^{(k+1)}}$. Since $k+1-s_{i q}^{(k+1)} \geq 1$ for $q=1,2, \cdots, k$, we thus have that $2^{k}=\sum_{q=1}^{k-1} 2^{k-s_{i_{q}}^{(k+1)}}+2^{k+1-s_{i k}^{(k+1)}}=\sum_{q=1}^{k-1} 2^{k-s_{i q}^{(k+1)}}+2^{k-\left(s_{i k}^{(k+1)}-1\right)}$.

Hence, after a suitable rearrangement of the entries, $\left[s_{i 1}^{(k+1)}, \cdots, s_{i, k-1}^{(k+1)}\right.$, $\left.s_{i k}^{(k+1)}-1\right]$ will be a row in $M_{k}$, that is, $\left[s_{i 1}^{(k+1)}, \cdots, s_{i, k-1}^{(k+1)}, s_{i k}^{(k+1)}-1\right]=\left[s_{p \sigma(1)}^{(k)}, \cdots s_{p, \sigma(k-1)}^{(k)}, s_{p, \sigma(k)}^{(k)}\right]$ for some $p$ and some permutation $\sigma$ on the set $\{1,2, \cdots, k\}$.

By noting that $s_{i k}^{(k+1)}=s_{p, \sigma(k)}^{(k)}+1$ and $s_{i k}^{(k+1)}=s_{i, k+1}^{(k+1)}$, we can thus conclude that $r_{i}$ is obtained from the $p^{t h}-$ row of $M_{k}$ by replacing the entry $s_{p, \sigma(k)}^{(k)}$ by the matrix $\left[s_{p, \sigma(k)}^{(k)}+1, s_{p, \sigma(k)}^{(k)}+1\right]$ and followed by a suitable rearrangement of the entries.

As a result of Corollary 4 and Theorem 5, we can easily exhibit the matrices $M_{k}$. For example, $M_{2}=[1,1], M_{3}=[1,2,2], M_{4}=\left(\begin{array}{cccc}1 & 2 & 3 & 3 \\ 2 & 2 & 2 & 2\end{array}\right)$, and $M_{5}=\left(\begin{array}{ccccc}1 & 2 & 3 & 4 & 4 \\ 1 & 3 & 3 & 3 & 3 \\ 2 & 2 & 2 & 3 & 3\end{array}\right)$.

LEMMA 6. Let each of $m, m^{\prime}$ and $t$ denote a positive integer. Then $2^{m} \equiv 2^{m^{\prime}} \bmod \left(2^{t}-1\right)$ if and only if $m \equiv m^{\prime} \bmod t$.

PROOF. Assume $m \geq m^{\prime}$. Suppose $m \equiv m^{\prime} \bmod t$. Then, $m=m^{\prime}+k t$ for some integer $k \geq 0$. Thus $2^{m}-2^{m^{\prime}}=2^{m^{\prime}+k t}-2^{m^{\prime}}=2^{m^{\prime}}\left(2^{k t}-1\right)$. Now $2^{t}-1 \mid 2^{k t}-1$ and so $2^{m} \equiv 2^{m^{\prime}} \bmod \left(2^{t}-1\right)$. Conversely, suppose $2^{m} \equiv 2^{m^{\prime}} \bmod \left(2^{t}-1\right)$. Then $2^{t}-1 \mid 2^{m}-2^{m^{\prime}}=2^{m^{\prime}}\left(2^{m-m^{\prime}}-1\right)$. Since gcd $\left(2^{t}-1,2^{m^{\prime}}\right)=1$, we thus have that $2^{t}-1 \mid 2^{m-m^{\prime}}-1$. Hence $t \mid m-m^{\prime}$ and therefore $m \equiv m^{\prime} \bmod t$.

THEOREM 7. Let $n=2^{m_{1}}+2^{m_{2}}+\cdots+2^{m_{k}}$, where $k \geq 2$, and each $m_{i}$ is a nonnegative integer. Also, let $t$ denote an integer $\geq 2$. Then $2^{t}-1 \mid n-1$ if and only if

$$
t \mid g c d\left(m_{\sigma(1)}+s_{i 1}^{(k)}, m_{\sigma(2)}+s_{i 2}^{(k)}, \cdots, m_{\sigma(k)}+s_{i k}^{(k)}\right)
$$

for some $\mathrm{i}$ and some permutation $\sigma$ on the set $\{1,2, \cdots, k\}$, where $\left[s_{i 1}^{(k)}, s_{\imath 2}^{(k)}, \cdots, s_{z k}^{(k)}\right]$ is the $i^{\text {th }}$ row of $M_{k}$.

PROOF. Suppose there exists a row $i$ in $M_{k}$ and a permutation $\sigma$ on the set $\{1,2, \cdots, k\}$ such that $\operatorname{gcd}\left(m_{\sigma(1)}+s_{i 1}^{(k)}, m_{\sigma(2)}+s_{i 2}^{(k)}, \cdots, m_{\sigma(k)}+s_{i k}^{(k)}\right)=d$ is divisible by the integer $t, t \geq$ 
2. Let $q$ denote a positive integer such that $q t \geq k$. Then $m_{\sigma(j)} \equiv q t-s_{i j}^{(k)} \bmod t$ for $j=$ $1,2, \cdots, k$. Hence, by Lemma 6 , we have that $n-1=2^{m_{\sigma(1)}}+2^{m_{\sigma(2)}}+\cdots+2^{m_{\sigma(k)}}-1 \equiv$ $2^{q t-s_{i 1}^{(k)}}+2^{q t-s_{i 2}^{(k)}}+\cdots+2^{g t-s_{i k}^{(k)}}-1 \bmod \left(2^{t}-1\right)$. Now, from the definition of the matrices $M_{k}$, we have that $2^{k-s_{i 1}^{(k)}}+2^{k-s_{i 2}^{(k)}}+\cdots+2^{k-s_{i k}^{(k)}}=2^{k}$. Multiplying both sides by $2^{g t-k}$ gives $2^{q t-s_{i 1}^{(k)}}+2^{q t-s_{i 2}^{(k)}}+\cdots+2^{q t-s_{i k}^{(k)}}=2^{q t}$. Hence $n-1 \equiv 2^{q t}-1 \bmod \left(2^{t}-1\right)$. Since $2^{t}-1 \mid 2^{q t}-1$, we thus have that $2^{t}-1 \mid n-1$.

Next, assume $2^{t}-1 \mid n-1$, where $t$ is an integer $\geq 2$. Also, suppose $n=2^{m_{1}}+2^{m_{2}}+\cdots+2^{m_{k}}$, where each $m_{i}$ is a nonnegative integer. We want to show that $t \mid g c d\left(m_{\sigma(1)}+s_{\imath 1}^{(k)}, \cdots, m_{\sigma(k)}+s_{1 k}^{(k)}\right)$ for some permutation $\sigma$ and some row $i$ of $M_{k}$. We will proceed by induction on $k$.

Suppose $k=2$ and $n=2^{m_{1}}+2^{m_{2}}$. Let $m_{1} \equiv m_{1}^{\prime} \bmod t$ and $m_{2} \equiv m_{2}^{\prime} \bmod t$, where $0 \leq$ $m_{1}^{\prime}, m_{2}^{\prime} \leq t-1$. Then, by Lemma $6, n \equiv 2^{m_{1}^{\prime}}+2^{m_{2}^{\prime}} \bmod \left(2^{t}-1\right)$. If $m_{1}^{\prime} \neq m_{2}^{\prime}$, then $2^{m_{1}^{\prime}}+2^{m_{2}^{\prime}}-1 \leq$ $2^{t-1}+2^{t-2}-1<2^{t-1}+2^{t-1}-1=2^{t}-1$ and this is a contradiction since $2^{t}-1 \mid 2^{m_{1}^{t}}+2^{m_{2}^{\prime}}-1$. Hence $m_{1}^{\prime}=m_{2}^{\prime}$ and $0 \equiv n-1 \equiv 2^{m_{1}^{\prime}+1}-1 \bmod \left(2^{t}-1\right)$. Thus $2^{t}-1 \mid 2^{m_{1}^{\prime}+1}-1$ which implies that $t \mid m_{1}^{\prime}+1$. Since $1 \leq m_{1}^{\prime}+1 \leq t$, we can conclude that $m_{1}^{\prime}+1=t$. Thus $m_{1}^{\prime}=m_{2}^{\prime}=t-1$ and consequently $m_{1}+1 \equiv m_{2}+1 \equiv 0 \bmod t$. Therefore $t \mid g c d\left(m_{1}+1, m_{2}+1\right)=g c d\left(m_{1}+s_{11}^{(2)}, m_{2}+s_{12}^{(2)}\right)$ from the definition of $M_{2}$.

Now suppose $k>2, n=2^{m_{1}}+2^{m_{2}}+\cdots+2^{m_{k}}$, and $2^{t}-1 \mid n-1$, where $t \geq 2$. Let $m_{i} \equiv m_{i}^{\prime} \bmod t$ for $i=1,2, \cdots, k$, where $0 \leq m_{i}^{\prime} \leq t-1$. We claim that the numbers $m_{i}^{\prime}$ cannot be all distinct. For, suppose that they were all distinct. Then $2^{m_{1}^{\prime}}+2^{m_{2}^{\prime}}+\cdots+2^{m_{k}^{\prime}}-1 \leq 2^{t-1}+2^{t-2}+\cdots+$

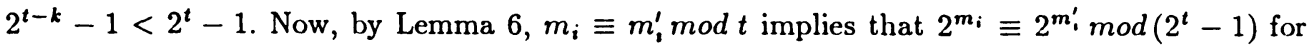
$i=1,2, \cdots, k$. Thus $2^{t}-1 \mid 2^{m_{1}^{\prime}}+2^{m_{2}^{\prime}}+\cdots+2^{m_{k}^{\prime}}-1$ and this contradicts the above statement that $2^{m_{1}^{\prime}}+2^{m_{2}^{\prime}}+\cdots+2^{m_{k}^{\prime}}-1<2^{t}-1$. Hence there exists an $\ell$ such that $m_{\ell}^{\prime}=m_{\ell+1}^{\prime}$. For convenience, we will assume $\ell=k-1$. Then $2^{m_{1}^{\prime}}+2^{m_{2}^{\prime}}+\cdots+2^{m_{k}^{\prime}}-1=2^{m_{1}^{\prime}}+2^{m_{2}^{\prime}}+\cdots+2^{m_{k-2}^{\prime}}+$ $2^{m_{k-1}^{\prime}+1}-1$. Now, by the induction hypothesis there is a pemutation $\sigma$ on the set $\{1,2, \cdots, k-$ 1) and a row $i$ in $M_{k-1}$ such that $t \mid g c d\left(m_{\sigma(1)}^{\prime}+s_{i 1}^{(k-1)}, \cdots, m_{\sigma(j)}^{\prime}+1+s_{i j}^{(k-1)}, \cdots, m_{\sigma(k-1)}^{\prime}+\right.$ $\left.s_{i, k-1}^{(k-1)}\right)$ where $\sigma(j)=k-1$. Since $m_{\sigma(1)} \equiv m_{\sigma(1)}^{\prime} \bmod t, \cdots, m_{\sigma(k-1)} \equiv m_{\sigma(k-1)}^{\prime} \bmod t$, we thus see that $t \mid g c d\left(m_{\sigma(1)}+s_{i 1}^{(k-1)}, \cdots, m_{\sigma(j)}+1+s_{i j}^{(k-1)}, \cdots, m_{\sigma(k-1)}+s_{i, k-1}^{(k-1)}\right)$ Now recalling that $m_{k-1}^{\prime}=m_{k}^{\prime}$ implies that $m_{k-1}+1+s_{i j}^{(k-1)} \equiv m_{k}+1+s_{i j}^{(k-1)} \bmod t$, we finally obtain that $t \mid g c d\left(m_{\sigma(1)}+s_{i 1}^{(k-1)}, \cdots, m_{k-1}+1+s_{i j}^{(k-1)}, m_{k}+1+s_{i j}^{(k-1)}, \cdots, m_{\sigma(k-1)}+s_{i, k-1}^{(k-1)}\right)$. This completes the proof since $\left\{m_{\sigma(1)}, \cdots, m_{k-1}, m_{k}, \cdots, m_{\sigma(k-1)}\right\}$ is a rearrangement of $\left\{m_{1}, m_{2}, \cdots, m_{k}\right\}$ and $\left[s_{i 1}^{(k-1)}, \cdots, s_{i, j-1}^{(k-1)}, 1+s_{i j}^{(k-1)}, 1+s_{i j}^{(k-1)}, s_{i, j+1}^{(k-1)}, \cdots, s_{i, k-1}^{(k-1)}\right]$ is, on being rearranged in ascending order, a certain row of the matrix $M_{k}$ which is obtained from the $i^{\text {th }}$ row of $M_{k-1}$ by replacing the $j^{\text {th }}$ entry by the $1 \times 2$ matrix $\left[1+s_{i j}^{(k-1)}, 1+s_{i j}^{(k-1)}\right]$.

4. MAIN RESULT.

THEOREM 8. Let $k$ denote an integer $\geq 2$ and let $n=2^{m_{1}}+2^{m_{2}}+\cdots+2^{m_{k}}$, where each $m_{i}$ is a nonnegative integer. Also, let $C$ denote the set such that $R \in C$ if and only if $R$ is a ring of characteristic two and $x^{n}=x$ for each $x \in R$. Then the following two statements are equivalent.

1) Each member in $C$ is boolean.

2) $\operatorname{gcd}\left(m_{\sigma(1)}+s_{i 1}^{(k)}, m_{\sigma(2)}+s_{i 2}^{(k)}, \cdots, m_{\sigma(k)}+s_{i k}^{(k)}\right)=1$ for each permutation $\sigma$ on the set $\{1,2, \cdots, k\}$ and for each row $\left[s_{i 1}^{(k)}, s_{i 2}^{(k)}, \cdots, s_{i k}^{(k)}\right]$ in $M_{k}$.

PROOF. Suppose each member in $C$ is boolean. Then, by Theorem 1 , there does not exist an integer $t \geq 2$ such that $2^{t}-1 \mid n-1$. Hence, by Theorem 7 , if $t \geq 2$, then $t \nmid \operatorname{gcd}\left(m_{\sigma(1)}+\right.$ $\left.s_{i 1}^{(k)}, \cdots, m_{\sigma(k)}+s_{i k}^{(k)}\right)$ for each $\sigma$ and each $i$. Thus $g c d\left(m_{\sigma(1)}+s_{i 1}^{(k)}, \cdots, m_{\sigma(k)}+s_{i k}^{(k)}\right)=1$ for each $i$ and $\sigma$.

Conversely, if $g c d\left(m_{\sigma(1)}+s_{i 1}^{(k)}, \cdots, m_{\sigma(k)}+s_{i k}^{(k)}\right)=1$ for each $i$ and $\sigma$, then, by Theorem 7 , 
$2^{t}-1 \nmid n-1$ for each integer $t \geq 2$. Hence, by Theorem $1, R$ is boolean for each $R \in C$.

5. EXAMPLES ILLUSTRATING THEOREM 8.

LEMMA 9. Let $R$ denote a ring and let $x \in R$ such that $x^{n}=x$ for some integer $n \geq 2$. If each of $h$ and $k$ is a positive integer such that $h \equiv k \bmod (n-1)$, then $x^{h}=x^{k}$.

This result can be obtained easily by induction, see [1].

LEMMA 10. Let $R$ denote a J-ring of characteristic two and suppose $n$ is a positive integer $\geq 2$. The following two statements are equivalent.

1) $x^{n}=x$ for each $x \in R$.

2) $x^{2 n-1}=x$ for each $x \in R$.

PROOF. Suppose (1) holds. Then (2) is immediate by Lemma 9 . Next, suppose $x^{2 n-1}=x$ for each $x \in R$. Then $x^{2 n}=x^{2}$ and thus $\left(x+x^{n}\right)^{2}=x^{2}+x^{2 n}=0$ since $R$ is of characteristic two. Hence $x^{n}=x$ since a J-ring does not contain non-zero nilpotent elements.

THEOREM 11. Let $R$ denote a ring of characteristic two. Suppose each of $s$ and $t$ is a positive integer with $s \neq t$ and $\operatorname{gcd}(s, t)=1$. If $x=x^{2^{s}}=x^{2^{t}}$ for each $x \in R$, then $R$ is boolean.

PROOF. We may assume that $s>1$. Then $x=x^{2^{s}}=x x^{2^{s}-1}=x^{2}{ }^{t} x^{2^{s}-1}=x^{2^{t}+2^{s}-1}=$ $x^{2\left(2^{t-1}+2^{s-1}\right)-1}$ for each $x \in R$. Hence, by Lemma 10, $x=x^{2^{t-1}+2^{s-1}}$ for each $x \in R$. Now $M_{2}=[1,1]$ and $\operatorname{gcd}(t-1+1, s-1+1)=\operatorname{gcd}(t, s)=1$. Consequently, by Theorem 8 , we have that $R$ is boolean.

The following examples illustrate the use of some of the preceding theorems.

EXAMPLE 1. Let $R$ denote a ring of characteristic two such that $x^{595}=x$ for each $x \in R$. Since $595=2(298)-1$ and $x^{595}=x$ for each $x \in R$ is equivalent, by Lemma 10 , to $x^{298}=x$ for each $x \in R$, we can thus apply our results to $x^{298}=x$. Now $298=2^{1}+2^{8}+2^{5}+2^{3}$. Using the matrix $M_{4}$ and applying Theorem 8 , we obtain $\operatorname{gcd}\left(1+s_{11}^{(4)}, 8+s_{12}^{(4)}, 5+s_{13}^{(4)}, 3+s_{14}^{(4)}\right)=$ $\operatorname{gcd}(1+1,8+2,5+3,3+3)=\operatorname{gcd}(2,10,8,6)=2 \neq 1$. Hence $R$ is not necessarily boolean.

EXAMPLE 2. Let $m$ denote a nonnegative integer. Let $R$ denote a ring of characteristic two and suppose $x^{n}=x$ for each $x \in R$, where $n=2^{m}+2^{m+1}+2^{m+2}$. Take $M_{3}=[1,2,2]$. Now $\operatorname{gcd}(m+1, m+1+2, m+2+2)=1, \operatorname{gcd}(m+2+1, m+1+2, m+2)=1$, and $\operatorname{gcd}(m+1+1, m+$ $2+2, m+2)=\operatorname{gcd}(m+2, m+4)$. Thus, by Theorem $8, R$ is boolean if $g c d(m+2, m+4)=1$, that is, if $m$ is odd, and not necessarily boolean if $m$ is even.

\section{REFERENCES}

1. AYOUB, R. and AYOUB, C. On commutativity of rings, Amer. Math. Monthly, $\underline{11}$, (1964), 267-271.

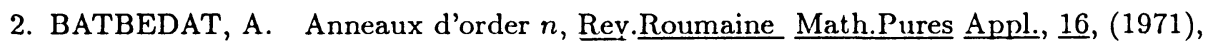
1305-1311.

3. JACOBSON, N. Structure of rings, Amer. Math. Soc. Colloq. Publ., New York, $\underline{37}$, (1956).

4. MACHALE, D. A remark on boolean rings, Mathematics Magazine, $\underline{63}$, (1990), 248-249.

5. SHIUE, J. S. and CHAO, W.M. On boolean rings, Yokohama Math. J., 24, (1976), 93-96. 


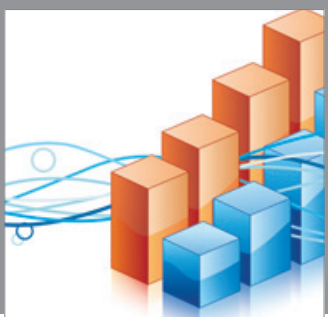

Advances in

Operations Research

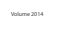

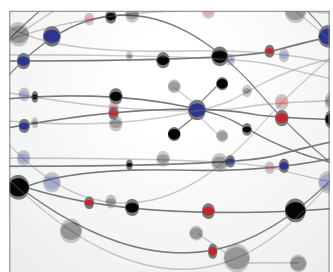

\section{The Scientific} World Journal
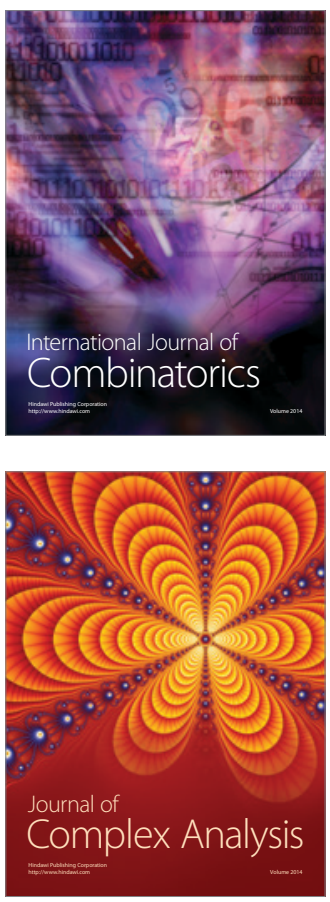

International Journal of

Mathematics and

Mathematical

Sciences
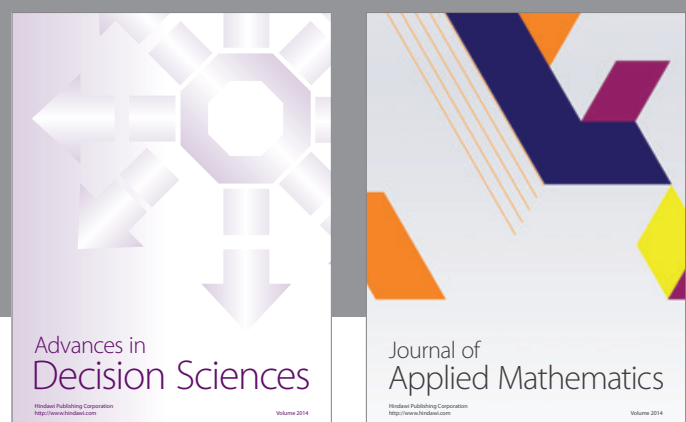

Journal of

Applied Mathematics
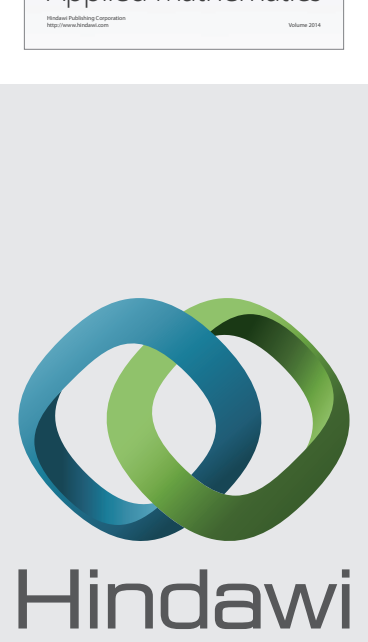

Submit your manuscripts at http://www.hindawi.com
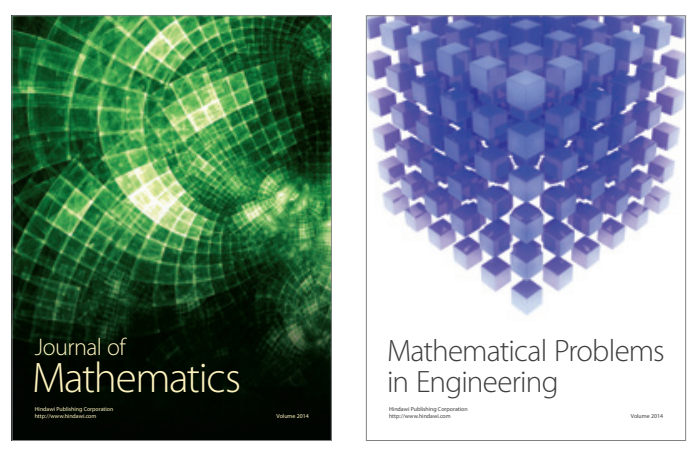

Mathematical Problems in Engineering
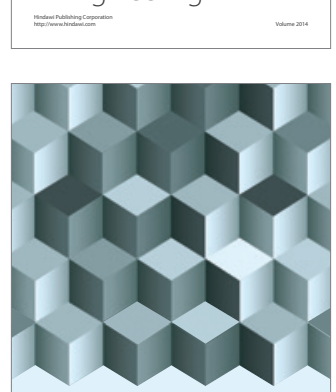

Journal of

Function Spaces
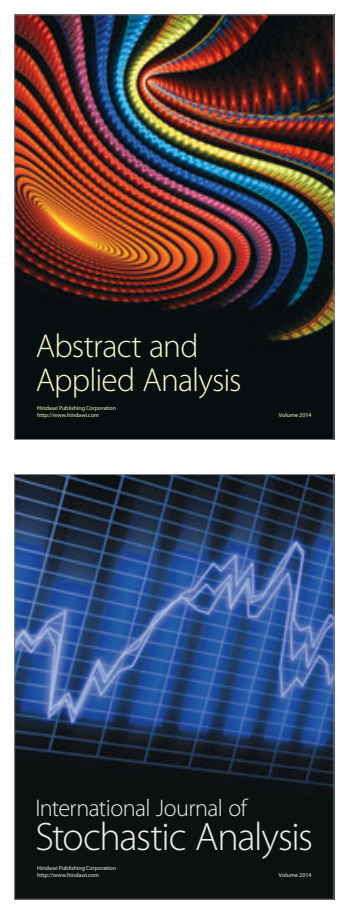

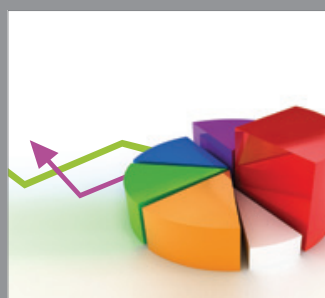

ournal of

Probability and Statistics

Promensencen
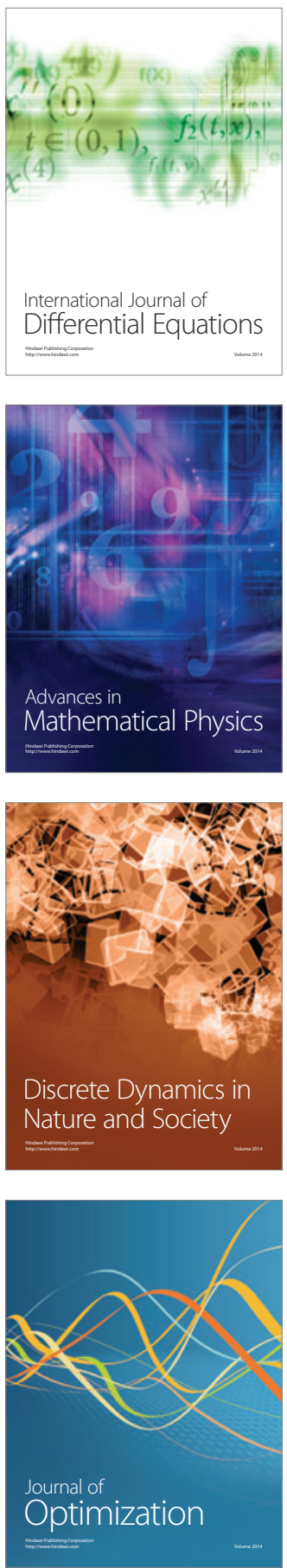